\title{
Myxomycetes associados a cactáceas no agreste e sertão de Pernambuco, Brasil
}

\author{
Inaldo do Nascimento Ferreira ${ }^{1 *}$ \\ Laise de Holanda Cavalcanti ${ }^{2}$ \\ ${ }^{1}$ Programa de Pós-Graduação em Biologia de Fungos, Centro de Ciências Biológicas \\ Departamento de Micologia, Universidade Federal de Pernambuco \\ Av. Prof. Moraes Rego s.n., CEP 50670-901, Recife - PE, Brasil \\ ${ }^{2}$ Laboratório de Myxomycetes, Departamento de Botânica \\ Centro de Ciências Biológicas, Universidade Federal de Pernambuco \\ *Autor para correspondência \\ inaldoferreira1@yahoo.com.br
}

Submetido em 25/11/2010

Aceito para publicação em 11/04/2011

\section{Resumo}

Os trabalhos que mencionam a presença de Myxomycetes em regiões áridas e semi-áridas do planeta têm revelado elevada diversidade de espécies e descrito novos táxons. Nestas regiões, os mixomicetos ocupam microhábitats especiais, como aqueles oferecidos por plantas suculentas. Com o objetivo de ampliar o conhecimento sobre as espécies que ocorrem em ambientes semi-áridos, foi investigada a presença de mixomicetos em Cereus jamacaru (mandacaru), Melocactus bahiensis (coroa de frade), Opuntia ficus-indica (palma forrageira) e Pilocereus gounellei (xique-xique), cactáceas que compõem a paisagem natural do agreste e sertão de Pernambuco, Brasil. Foram obtidos 158 espécimes (106 coletados em campo; 52 em câmara-úmida), representando 16 espécies, distribuídas em nove gêneros, com elevada diversidade taxonômica $(\mathrm{S} / \mathrm{G}=0,63-1,5)$. Todas as espécies são novas referências para os três municípios estudados. Comatricha pulchella, Didymium nigripes e Physarum bogoriense são mencionadas pela primeira vez para a Caatinga no estado de Pernambuco e Didymium squamulosum e Physarum echinosporum são novas referências para este bioma. Badhamia melanospora e Physarum compressum foram as espécies mais abundantes e frequentes, caracterizando-se como constantes na mixobiota estudada.

Palavras-chave: Caatinga, Microhábitat, Mixobiota, Semi-árido, Suculentas

\section{Abstract}

Myxomycetes associated with Cactaceae in sub-humid (agreste) and semi-arid (sertão) regions of Pernambuco, Brazil. Studies that indicate the presence of Myxomycetes in arid and semi-arid regions of the planet have revealed new taxons and high species diversity. In those environments, the Myxomycetes occupy special microhabitats like those offered by succulent plants. Aiming to expand the knowledge of species that occur in semi-arid environments we investigated the occurrence of Myxomycetes on Cereus jamacaru (mandacaru), Melocactus bahiensis (coroa de frade), Opuntia ficus-indica (palma forrageira) and Pilocereus gounellei (xique-xique) - cacti that compose the natural landscape of the sub-humid (agreste) and semi-arid (sertão) regions of Pernambuco, Brazil. We obtained 158 specimens of Myxomycetes (106 field collections; 52 in moist chamber cultures), representing 16 species distributed in nine genera, with high taxonomic diversity (S/ 
$\mathrm{G}=0.63-1.5$ ). All of the identified species are new records for the municipalities studied. Comatricha pulchella, Didymium nigripes and Physarum bogoriense are mentioned for the first time in Caatinga areas in the state of Pernambuco, and Didymium squamulosum and Physarum echinosporum are new records for this biome. Physarum compressum and Badhamia melanospora were the most abundant and frequent species, characterized as constant in the studied myxobiota.

Key words: Caatinga biome, Microhabitat, Myxobiota, Semi-arid, Succulent plants

\section{Introdução}

Os poucos inventários feitos para as regiões desérticas e semidesérticas, em diferentes continentes, evidenciam que a possibilidade de algumas espécies desenvolver-se em ambientes com elevada restrição hídrica existe em quase todas as ordens de Myxomycetes, como se pode verificar nas pesquisas realizadas por Schnittler (2001) e Cavalcanti et al. (2006). Estes e outros estudos também revelaram uma alta diversidade e riqueza de espécies de Myxomycetes em desertos quentes ou frios de diferentes países (MOSQUERA et al., 2000; 2003; SCHNITTLER; NOVOZHILOV, 2000; STEPHENSON et al., 2000; STEPHENSON et al., 2004; LADO et al., 2006; NOVOZHILOV et al., 2006; 2008).

No Brasil, os estudos sobre a mixobiota presente em ambientes semi-áridos foram iniciados por Gottsberger (1968), que mencionou oito espécies para áreas de Caatinga, coletadas na Chapada Diamantina, Bahia. Neste trabalho, foi descrita Didymium bahiense Gottsb., cujo material tipo foi coletado sobre planta suculenta da Caatinga do Morro do Chapéu. O inventário feito recentemente por Cavalcanti et al. (2006) revelou a ocorrência de 95 espécies de mixomicetos para diversos municípios situados na região semi-árida do Nordeste, porém muitas delas foram coletadas nas ilhas de floresta úmida conhecidas como brejos de altitude, encravadas na Caatinga.

O bioma Caatinga cobre uma área com cerca de $800.000 \mathrm{~km}^{2}$, que se estende pela maior parte da região Nordeste até o norte de Minas Gerais (SAMPAIO et al., 2002). A vegetação tem fisionomia típica das zonas semi-áridas, determinada pelas temperaturas médias elevadas, índices pluviométricos muito baixos e chuvas concentradas em curtos períodos do ano. As plantas apresentam adaptações ao clima, com folhas ausentes ou com cutículas altamente impermeáveis, caules suculentos e fotossintetizantes, sendo frequentes as cactáceas, agaváceas e bromeliáceas (JATOBÁ, 2003; AB'SABER, 2008).

Apesar destas características a Caatinga possui uma grande diversidade de plantas e animais, com níveis elevados de endemismo, e parte significativa do seu patrimônio biológico não é encontrada em qualquer outro lugar do mundo (PINHEIRO; ALVES, 2007; AB'SABER, 2008). Diversos estudos sobre a macroflora e macrofauna já foram realizados (LEAL et al., 2003; RODAL et al., 2008; 2009), porém são poucas as informações sobre fungos e mixomicetos, principalmente as referentes aos aspectos ecológicos (GUSMÃO; MAIA, 2006).

No presente trabalho investigou-se a ocorrência e distribuição de mixomicetos associados com espécies de Cactaceae em áreas do agreste e do sertão pernambucano, visando ampliar o conhecimento sobre esses organismos em ambientes semi-áridos.

\section{Material e Métodos}

\section{Áreas de estudo}

$\mathrm{O}$ estudo foi realizado em áreas de três municípios localizados entre as mesorregiões do agreste e do sertão pernambucano onde estão presentes espécies pertencentes à família Cactaceae, representadas por Cereus jamacaru DC., o mandacaru, Melocactus bahiensis (Britton et Rose) Luetzelb., conhecido como coroa de frade, Opuntia ficus-indica Mill., a palma forrageira e Pilocereus gounellei F.A.C Weber ex K. Schum., o xique-xique (Figura 1).

Como critério, para cada município referido, foi selecionada uma área onde a vegetação nativa estava aparentemente bem preservada ou correspondiam a uma Unidade de Conservação. 

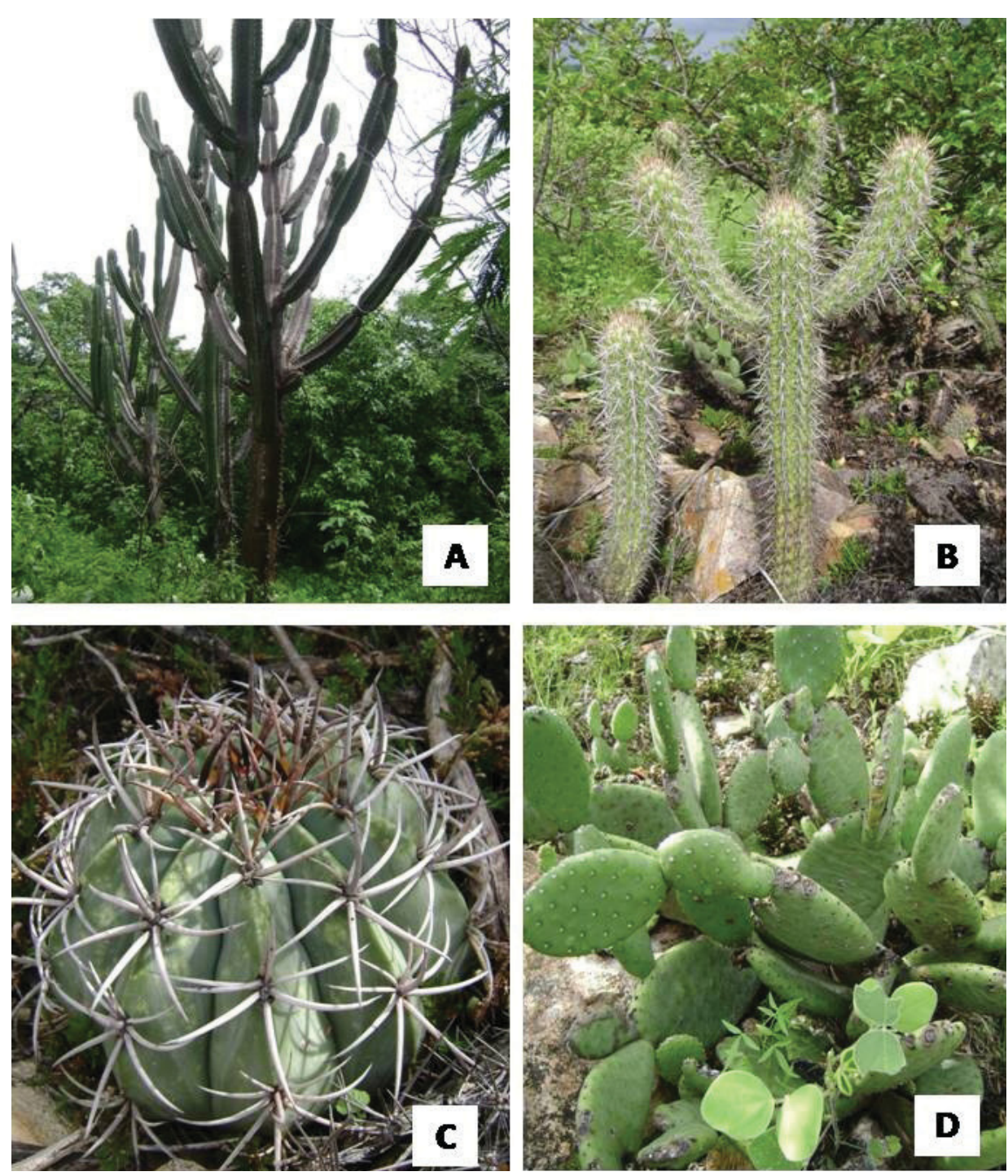

FIGURA 1: A. Mandacaru (Cereus jamacaru DC); B. Xiquexique (Pilocereus gounellei F.A.C Weber ex K. Schum.); C. Coroa de frade (Melocactus bahiensis (Britton et Rose) Luetzelb.; D. Palma forrageira (Opuntia ficus-indica Mill.).

Município de Buíque - Parque Nacional do Catimbau (37ㅇ' $\mathrm{W}$ e $8^{\circ} 37^{\prime} \mathrm{S}, 702 \mathrm{~m}$ alt., 62.500ha). Segundo a classificação de Köeppen o clima local é quente e seco (tipo BShs'), típico do semi-árido brasileiro, com temperatura média anual de $26^{\circ} \mathrm{C}$ e precipitação média anual de $600 \mathrm{~mm}$, com a estação chuvosa ocorrendo entre os meses de abril e junho (SUDENE, 1990). Localizado na zona de transição entre Agreste e Sertão, a área estudada apresenta vegetação de Caatinga hiperxerófila e hipoxerófila.

Município de Serra Talhada - Estação Experimental do Instituto de Pesquisas Agropecuárias - IPA da Empresa Pernambucana de Pesquisa Agropecuária
(7054'701'S e $38^{\circ} 17^{\prime} 495^{\prime \prime} \mathrm{W}, 513 \mathrm{~m}$ alt., 3.200ha). O clima é quente e semi-árido (Köeppen BShw'), com chuvas escassas e mal distribuídas, com períodos de seca entre abril e novembro. As temperaturas médias chegam a $25^{\circ} \mathrm{C}$ com precipitação média de $700 \mathrm{~mm}$. Localizado na Mesorregião do sertão pernambucano, semi-árido nordestino, a área apresenta vegetação de Caatinga hiperxerófila arbórea-arbustiva.

Município de Mirandiba - Neste município foi selecionada uma área bem preservada de Caatinga hiperxerófila arbóreo-arbustiva ( $8^{\circ} 7^{\prime} 902^{\prime} \mathrm{S}$ e 3843'529"W, 465m alt., 50ha.), Localizado na Mesorregião do sertão pernambucano, semi-árido 
nordestino, o clima do município é quente e semiárido (Köeppen BShw'), com chuvas escassas e mal distribuídas, com períodos de seca entre abril e novembro. Temperatura média de $25,2^{\circ} \mathrm{C}$ e precipitação média de 600mm (PINHEIRO; ALVES, 2007).

\section{Procedimento de campo e de laboratório}

Em cada área três coletas foram efetuadas, em dois anos consecutivos (2007 - 2008), durante os períodos chuvoso e de estiagem. Em cada local foram exploradas trilhas com extensão média de $1 \mathrm{~km}$, onde foi pesquisada a presença de mixomicetos em cactáceas. Também foi coletado material para montagem de 400 câmaras-úmidas (SCHNITTLER; STEPHENSON, 2002). Para identificação das espécies foi utilizada literatura especializada (LISTER, 1925; MARTIN; ALEXOPOULOS, 1969; FARR, 1976; LADO; PANDO 1997). Foi seguido o sistema de classificação de Martin et al. (1983) para gêneros e categorias supragenéricas e Lado (2001) para a nomenclatura e a abreviatura dos nomes dos autores das espécies. Foi considerado como espécime um ou o conjunto de mais esporocarpos originados de um mesmo plasmódio, como descrito por Stephenson (1989).

Para aquelas espécies com elevado número de espécimes foi selecionado o material mais representativo do táxon para ser citado como material estudado no texto e para depósito no Herbário UFP da Universidade Federal de Pernambuco. O índice de diversidade taxonômica ( $\mathrm{S} / \mathrm{G}=$ Espécies/gêneros) foi estimado para a mixobiota encontrada nas quatro cactáceas estudadas (STEPHENSON et al., 1993).

A sazonalidade das espécies foi relacionada à presença/ausência de esporocarpos, considerando-se também o número de espécimes obtidos nos períodos de estiagem e chuvoso, para observar picos de esporulação. Foram avaliadas a abundância e constância das espécies, como utilizadas por Schnittler et al. (2002) e Cavalcanti e Mobin (2004).

Abundância - escassa $(<1,5 \%)$, ocasional $(1,5$ $3,5 \%)$, comum $(>3,5-6,5 \%)$, abundante $(>6,5 \%)$.
Constância - acidental $(<25 \%)$, acessória (25$50 \%)$, constante $(>50 \%)$.

\section{Resultados e Discussão}

Foram obtidos 106 espécimes no campo e 52 esporularam em câmara-úmida, tendo como substrato as quatro espécies de cactos selecionadas para estudo. Destes materiais, 16 espécies foram identificadas, distribuídas em nove gêneros, cinco famílias e quatro ordens, representando as três subclasses de Myxomycetes.

\section{Lista comentada das espécies}

\section{Ceratiomyxomycetidae}

\section{Ceratiomyxales}

\section{Ceratiomyxaceae}

Ceratiomyxa fruticulosa (O. F. Müll.) T. Macbr., N. Amer. Slime-Moulds 18, 1899.

Material examinado: BRASIL, Pernambuco, Serra Talhada, Estação Experimental da Empresa Pernambucana de Pesquisa Agropecuária - IPA, Ferreira et al. 12, 02/VI/2007 (UFP 48237); ibid, Ferreira et al. 13, 02/VI/2007 (UFP 48238); ibid, Ferreira et al. 125, 26/VII/2008 (UFP 54356).

Comentários: três espécimes foram coletados durante as excursões de campo, esporulando no interior de cladódios mortos de mandacaru. Ceratiomyxa fruticulosa apresenta estruturas muito frágeis e que sofrem rápida dessecação, esporulando em cactos em decomposição cujos tecidos apresentavam ainda um bom teor de água.

Ceratiomyxa fruticulosa tem uma ampla distribuição no semi-árido brasileiro, para o qual foi mencionada pela primeira vez por Gottsberger (1968), com base em coletas no município de Gameleira dos Crentes (BA). Foi também mencionada por Cavalcanti et al. (2006) e Alves et al. (2010) para os municípios de Caetité e Morro do Chapéu (BA), Crato e Pacujá (CE) e Piripiri (PI). Porém no Crato e em Piripiri os espécimes foram coletados em fragmento de floresta úmida, inserido no bioma Caatinga. Considerando a constância e abundância nos substratos analisados, 
Ceratiomyxa fruticulosa foi classificada como acessória e ocasional, respectivamente, esporulando no período de estiagem (Tabela 1).

\section{Myxogastromycetidae}

\section{Trichiales}

\section{Trichiaceae}

Arcyria cinerea (Bull.) Pers., Syn. Meth. Fung.: 184, 1801.

Material examinado: BRASIL, Pernambuco, Serra Talhada, Estação Experimental da Empresa Pernambucana de Pesquisa Agropecuária - IPA, Ferreira et al. 139, 27/VII/2008 (UFP 54360); ibid, Ferreira et al. 140, 27/VII/2008 (UFP 54359).

Comentários: apenas dois espécimes foram obtidos durante o presente estudo, ambos no período de estiagem sendo os esporocarpos encontrados em mandacaru e palma forrageira.

Espécie bastante comum em diversos ecossistemas e substratos, Arcyria cinerea possui ampla distribuição na região semi-árida do Brasil. De acordo com Cavalcanti et al. (2006); Gusmão et al. (2005) e Alves et al. (2010) essa espécie ocorre nos municípios baianos de Jussiape, Lençóis e Morro do Chapéu, na Chapada Diamantina, bem como no Crato, Graça e Pacujá (CE), Piripiri (PI), Caruaru e São Caetano (PE). No presente estudo, a espécie foi considerada como acidental e escassa, com registro em apenas um dos três municípios estudados (Tabela 1).

Hemitrichia calyculata (Speg.) M.L. Farr, Mycologia 66(5): 887, 1974.

Material examinado: BRASIL, Pernambuco, Serra Talhada, Estação Experimental da Empresa Pernambucana de Pesquisa Agropecuária - IPA, Ferreira et al. 152, 27/VII/2008 (UFP 54445).

Comentários: apenas um espécime foi obtido, coletado no período de estiagem, na parte externa de cladódios mortos de mandacaru. Para o semi-árido brasileiro Hemitrichia calyculata apresenta registros para os municípios de Lençóis (BA), Crato, Graça, Ibiapina e Pacujá (CE), Brejo da Madre de Deus e Triunfo (PE) e Piripiri (PI), segundo Gusmão et al.
(2005), Cavalcanti et al. (2006) e Alves et al. (2010); com exceção de Lençóis, Graça e Pacujá, os locais de coleta são brejos de altitude e em Piripiri, no Parque Nacional de Sete Cidades, a espécie ocorreu em ambiente de floresta secundária, mata ciliar e cerrado. Apesar de comumente encontrada em diversos ecossistemas do Brasil, inclusive no bioma Caatinga, no presente estudo Hemitrichia calyculata mostrou-se acidental e escassa (Tabela 1).

Hemitrichia serpula (Scop.) Rostaf. ex Lister, Monogr. Mycetozoa: 179, 1894.

Material examinado: BRASIL, Pernambuco, Serra Talhada, Estação Experimental da Empresa Pernambucana de Pesquisa Agropecuária - IPA, Ferreira et al. 141, 27/VII/2008 (UFP 55278).

Comentários: um único espécime foi coletado sobre mandacaru, apresentando somente restos do típico capilício, com filamentos ornamentados por espirais e longos espinhos, e esporo reticulado.

Hemitrichia serpula possui ampla distribuição no Brasil (BEZERRA et al., 2009), porém nas listas de Cavalcanti et al. (2006) e Alves et al. (2010) sua ocorrência foi constatada apenas nos municípios de Lençóis (BA), Graça, Itapipoca e Pacujá (CE). Considerando a constância e abundância nos substratos analisados Hemitrichia serpula mostrou-se acidental e escassa, esporulando na estiagem (Tabela 1).

Perichaena corticalis (Batsch) Rostaf., Sluzowce Monogr. 293, 1875.

Material examinado: BRASIL, Pernambuco, Serra Talhada, Estação Experimental da Empresa Pernambucana de Pesquisa Agropecuária - IPA, Ferreira et al. 15, 02/VI/2007 (UFP 54446); ibid, Ferreira et al. 16, 02/VI/2007 (UFP 52075); ibid, Ferreira et al. 17, 02/VI/2007 (UFP 54461); ibid, Ferreira et al. 57, 02/VI/2007 (UFP 54451); ibid, Ferreira et al. 68, 02/ VI/2007 (UFP 54447).

Comentários: no presente estudo cinco espécimes foram obtidos através do cultivo em câmara-úmida confeccionada com cladódios mortos de coroa-de-frade. Todos os esporocarpos se desenvolveram nos espinhos 
da planta. Apesar de ser citada para regiões áridas de outros países (ESTRADA-TORRES et al., 2009), no Brasil esta espécie foi registrada somente para os municípios de Jussiape (BA), em ambiente de caatinga arbórea densa, Bezerros e Garanhuns, no agreste de Pernambuco (GUSMÃO et al., 2005; CAVALCANTI et al., 2006). Considerando a constância e abundância nos substratos analisados, Perichaena corticalis mostrou-se acidental e ocasional na mixobiota estudada (Tabela 1).

Perichaena depressa Lib., P1. Crypt. Arduenna: 378, 1837.

Material examinado: BRASIL, Pernambuco, Serra Talhada, Estação Experimental da Empresa Pernambucana de Pesquisa Agropecuária - IPA, Ferreira et al. 131, 27/VII/2008 (UFP 54366); ibid, Ferreira et al. 132, 27/VII/2008 (UFP 54441); ibid, Ferreira et al. 142, 12/IV/2008 (UFP 54443).

Comentários: três espécimes, formando extensas frutificações, foram observados sobre cladódios mortos de mandacaru, no período de estiagem.

Perichaena depressa já foi referida para a região semi-árida do Brasil (GUSMÃO et al., 2005; CAVALCANTI et al., 2006; ALVES et al., 2010), com registros para os municípios de Jussiape (BA), Graça, Sobral e Crato (CE) e Brejo da Madre de Deus (PE), os dois últimos em encraves de floresta úmida na caatinga. No presente estudo a espécie mostrou-se ocasional e acidental, registrada em apenas um dos três municípios estudados (Tabela 1).

\section{Physarales}

\section{Physaraceae}

Physarum bogoriense Racib., Hedwigia 37: 52, 1898.

Material examinado: BRASIL, Pernambuco, Serra Talhada, Estação Experimental da Empresa Pernambucana de Pesquisa Agropecuária - IPA, Ferreira et al. 28, 11/IV/2008 (UFP 55274); ibid, Ferreira et al. 46, 13/IV/2008 (UFP 54453); Mirandiba, Ferreira et al. 04, 13/IV/2008, (UFP 56054).

Comentários: durante as coletas de campo, realizadas no período chuvoso, foram obtidos três espécimes esporulando sobre mandacaru. Physarum bogoriense é uma espécie tropical e foi encontrada pela primeira vez na Caatinga, no município de Gameleira dos Crentes (BA), por Gottsberger (1968). Os espécimes coletados em Serra Talhada constituem o primeiro registro da ocorrência da espécie em Caatinga pernambucana. Considerando a constância e abundância nos substratos analisados, Physarum bogoriense enquadrou-se como acessória e ocasional (Tabela 1).

Physarum compressum Alb. \& Schwein., Consp. Fung. Lusat.: 97, 1805.

Material examinado selecionado: BRASIL, Pernambuco, Serra Talhada, Estação Experimental da Empresa Pernambucana de Pesquisa Agropecuária IPA, Ferreira et al. 17, 13/IV/2008 (UFP 54462); ibid, Ferreira et al. 43, 12/IV/2008 (UFP 54455); ibid, Ferreira et al. 55, 02/VII/2008 (UFP 54468); ibid, Ferreira et al. 59, 01/VII/2007 (UFP 54460); ibid, Ferreira et al. 60, 02/VI/2008 (UFP 54457); ibid, Ferreira et al. 660, 2/VI/2007 (UFP 54459); ibid, Ferreira et al. 130, 26/ VII/2008 (UFP 54444). Mirandiba, Ferreira et al. 14, 13/IV/2008 (UFP 54461); ibid, Ferreira et al. 18, 13/ IV/2008 (UFP 54464).

Comentários: no presente estudo Physarum compressum representa cerca de 1/3 das coleções obtidas tanto em campo quanto em câmaras-úmidas; a espécie foi registrada em quase todos os meses de coleta, esporulando nas quatro cactáceas estudadas. Essa espécie é altamente associada a plantas suculentas, aparentemente desenvolvendo-se bem em um $\mathrm{pH}$ básico, o que ocorre em diversas cactáceas de regiões áridas de diferentes países e continentes, como o México, onde foi registrada sobre as suculentas Ferocatus latispinus (Haworth) Britton \& Rose, Myrtillocactus geometrizans (Mart. ex Pfeiff.) Console; Pachycereus weberi (J.M.Coult. ) Backeb. e Yucca periculosa Baker (ESTRADA-TORRES et al., 2009).

$\mathrm{Na}$ região semi-árida do país Physarum compressum foi registrada no município do Crato (CE) e nos municípios de Bezerros, Caruaru e Pesqueira, do agreste pernambucano por Cavalcanti et al. (2006). Considerando a constância e abundância nos substratos analisados, Physarum compressum mostrou-se constante e abundante, esporulando no período chuvoso e de estiagem (Tabela 1). 
Physarum echinosporum Lister, J. Bot. 37:147, 1899.

Material examinado: BRASIL, Pernambuco, Serra Talhada, Estação Experimental da Empresa Pernambucana de Pesquisa Agropecuária - IPA, Ferreira et al. 26, 11/IV/2008 (UFP 54362); ibid, Ferreira et al. 27, 12/IV/2008 (UFP 54442); ibid Ferreira et al. 28, 13/ IV/2008 (UFP 54458). Mirandiba, Ferreira et al. 11, 13/ IV/2008 (UFP 54363).

Comentários: todos os espécimes obtidos foram coletados diretamente no campo, durante os períodos chuvosos e de estiagem, formando sempre extensas frutificações no interior e exterior de cladódios mortos de mandacaru e palma forrageira (Tabela 1). A ocorrência de Physarum echinosporum é conhecida para os estados de Pernambuco, Paraíba e São Paulo (MAIMONIRODELLA, 2002; CAVALCANTI, 2002; COSTA et al., 2010), porém apenas para a Mata Atlântica, sendo este o primeiro registro para o bioma Caatinga. No presente estudo a espécie mostrou-se acessória e ocasional na mixobiota local (Tabela 1).

Physarum pusillum (Berk. \& M. A. Curtis) G. Lister, in Lister, Monogr. Mycetozoa, ed. 2 64, 1911.

Material examinado: BRASIL, Pernambuco, Serra Talhada, Estação Experimental da Empresa Pernambucana de Pesquisa Agropecuária - IPA, Ferreira et al. 15, 13/IV/2008 (UFP 54452); ibid, Ferreira et al. 16, 13/IV/2008 (UFP 54466); ibid, Ferreira et al. 23, 13/IV/2008 (UFP 54465); ibid, Ferreira et al. 15, 12/ IV/2008 (UFP 54450).

Comentários: quatro espécimes foram obtidos em ambiente natural, com os esporocarpos se desenvolvendo em espinhos de mandacaru e no interior de cladódios de palma forrageira e xiquexique. Na Caatinga, Physarum pusillum foi mencionada por Gottsberger (1968), para o município de Gameleira dos Crentes (BA), e, posteriormente, por Cavalcanti et al. (2006), para Águas Belas, Caruaru e Fazenda Nova, situados no sertão e agreste de Pernambuco. Considerando a constância e abundância nos substratos analisados, Physarum pusillum mostrou-se acidental e ocasional, esporulando na estiagem (Tabela 1).

Badhamia melanospora Speg., Anales Soc. Ci. Argent. 10:150, 1880.
Material examinado selecionado: BRASIL, Pernambuco, Serra Talhada, Estação Experimental da Empresa Pernambucana de Pesquisa Agropecuária - IPA, Ferreira et al. 42, 11/IV/2008 (UFP 54467), ibid, Ferreira et al. 13, 13/IV/2008 (UFP 54463); ibid, Ferreira et al. 18, 13/ IV/08 (UFP 54464); ibid, Ferreira et al. 44, 13/IV/2008 (UFP54454). Mirandiba, Ferreira et al. 19, 13/IV/2008 (UFP 54449); ibid, Ferreira et al. 20, 13/IV/2008 (UFP 54456); ibid, Ferreira et al. 42, 14/IV/2008 (UFP 54467); ibid, Ferreira et al. 33, 28/ VII/2008 (UFP 54467 ); ibid, Ferreira et al. 34, 13/ IV/2008 (UFP 54592). Buíque, Parque Nacional Vale do Catimbau, Bezerra et al. 30, 22/VIII/2006 (UFP 1366); ibid, Bezerra 32, 23/VIII/2006 (UFP 1368); ibid, Bezerra 37, 24/VIII/2006 (UFP 1367).

Comentários: os espécimes obtidos representam cerca da metade do material coletado durante o estudo em ambiente natural nas estações seca e chuvosa, e daqueles originados com a técnica do cultivo em câmaras-úmidas. Badhamia melanospora é comum e abundante nas quatro espécies de cactos escolhidas para estudo, esporulando nas partes internas e externas dos cladódios mortos, incluindo os espinhos das plantas.

Conforme dados da literatura esta espécie também é altamente associada às suculentas em diversas regiões áridas do planeta (NOVOZHILOV et al., 2003; LADO et al., 2006).

Badhamia melanospora é uma espécie com morfologia variável (CASTILLO et al., 1996) e esse fato foi também observado nas coleções obtidas em todos os locais onde foi realizado o presente estudo, registrando tanto esporângios sésseis quanto pedicelados. Até o momento sua presença foi registrada somente para áreas de Caatinga por Cavalcanti et al. (2006), no sertão (Águas Belas) e no agreste (Caruaru, Fazenda Nova e Pesqueira) de Pernambuco, e no município de Pacujá, noroeste do Ceará (ALVES et al., 2010). No presente estudo a espécie mostrou-se constante e abundante, presente em todos os municípios estudados (Tabela 1).

\section{Didymiaceae}

Diachea leucopodia (Bull.) Rostaf., Sluzowce Monogr. 190, 1874. 
Material examinado: BRASIL, Pernambuco, Mirandiba, Ferreira et al. 31, 28/VII/2008 (UFP 54364).

Comentários: um único registro foi efetuado no presente estudo, em ambiente natural sobre espinhos de xique-xique. Apesar de constar na lista das espécies citadas para a região semi-árida brasileira (CAVALCANTI et al., 2006) o registro corresponde ao ambiente de cerrado existente no Parque Nacional de Sete Cidades, no Piauí (CAVALCANTI e MOBIN, 2004). Embora ocorra nas estepes áridas e frias da bacia do Rio Volga (Rússia), esta espécie é mais comumente encontrada nos ambientes úmidos e quentes ou temperados dos dois hemisférios. No Brasil, Diachia leucopodia tem registros para as regiões Nordeste, Sudeste e Sul, em fragmentos de Floresta Atlântica e manguezais, com um único registro para a Caatinga, feito por Cavalcanti et al. (2009). Considerando a constância e abundância nos substratos analisados, Diachia leucopodia mostrou-se acidental e escassa, esporulando na estiagem apenas no município de Mirandiba (Tabela 1).

Didymium minus (Lister) Morgan, J. Cincinnati Soc. Nat. Hist. 16:145, 1894.

Material examinado: BRASIL, Pernambuco, Serra Talhada, Estação Experimental da Empresa Pernambucana de Pesquisa Agropecuária - IPA, Ferreira et al. 123, 27/VII/2008 (UFP 54440).

Comentários: um único espécime foi obtido durante as coletas de campo, esporulando no interior de cladódios de xique-xique em decomposição. Sua distribuição no semi-árido brasileiro era restrita ao estado da Bahia, mencionada para os municípios de Gameleira dos Crentes, Belo Campo e Morro do Chapéu (GOTTSBERGER, 1968; CAVALCANTI et al., 2006). Considerando a constância e abundância nos substratos analisados, Didymium minus mostrou-se acidental e escassa, esporulando na estiagem, ocorrendo apenas no município de Serra Talhada (Tabela 1).

Didymium nigripes (Link) Fr., Syst. Mycol. 3:119, 1829.

Material examinado: BRASIL, Pernambuco, Serra Talhada, Estação Experimental da Empresa Pernambucana de Pesquisa Agropecuária - IPA, Ferreira et al. 118, 27/VII/2008 (UFP 55288).

Comentários: um único espécime foi encontrado no período de estiagem, esporulando no interior de cladódios de xique-xique em decomposição (Tabela 1). Estrada-Torres et al. (2009) registraram pela primeira vez a ocorrência dessa espécie, tipicamente foliícola, sobre Opuntia sp. Apesar de citada por Cavalcanti et al. (2006), o registro foi efetuado em um encrave de Cerrado na região semi-árida do Brasil, no Parque Nacional de Sete Cidades (CAVALCANTI e MOBIN, 2004). Assim, esta é a primeira referência da espécie para áreas de vegetação típica de Caatinga em Pernambuco. No presente estudo a espécie mostrou-se acidental e escassa, presente apenas em um dos três municípios estudados (Tabela 1).

Didymium squamulosum (Alb. \& Schwein.) Fr., Symb. Gasteromyc. 19, 1818.

Material examinado: BRASIL, Pernambuco, Serra Talhada, Estação Experimental da Empresa Pernambucana de Pesquisa Agropecuária - IPA, Ferreira et al. 186, 27/VII/2008 (UFP 54593 ); ibid, Ferreira et al. 187, 27/VII/2008 (UFP 54594 ); ibid, Ferreira et al. 188, 27/VII/2008 (UFP 54595); ibid, Ferreira et al., 189, 27/VII/2008 (UFP 54596); ibid, Ferreira et al. 190, $27 /$ VII/2008 (UFP 54597).

Comentários: na forma típica, os esporocarpos de Didymium squamulosum apresentam geralmente um curto pedicelo, fortemente calcário. No presente estudo, as coleções obtidas apresentaram-se como plasmodiocarpos e foram identificadas como Didymium squamulosum com base nos comentários e ilustração de Lister (1925), que menciona esse tipo de esporocarpo. Extensas frutificações foram encontradas na parte externa dos cladódios mortos da palma forrageira. Beltrán-Tejera et al. (2010) referem a ocorrência de Didymium squamulosum sobre Opuntia maxima Miller, nas ilhas Canárias, porém são raros os registros desta espécie em ambientes áridos, sendo esta a primeira vez que é mencionada para a Caatinga. Considerando a constância e abundância nos substratos analisados, Didymium squamulosum mostrou-se acidental e ocasional, esporulando na estiagem (Tabela 1). 


\section{Stemonitomycetidae}

\section{Stemonitales}

\section{Stemonitaceae}

Comatricha pulchella (C. Bab.) Rostaf., Sluzowce Monogr. Suppl. 27, 1876.

Material examinado: BRASIL, Pernambuco, Serra Talhada, Estação Experimental da Empresa Pernambucana de Pesquisa Agropecuária - IPA, Ferreira et al. 190, 02/VI/2007 (UFP 54814).

Comentários: apenas um espécime foi obtido, esporulando em câmara-úmida montada com cladódios mortos de mandacaru. A presença da espécie foi anteriormente registrada em área de caatinga arbustiva no Morro do Chapéu, no semi-árido baiano (GUSMÃO et al., 2005; CAVALCANTI et al., 2006), sendo a coleção deste trabalho a primeira referência da espécie para áreas de Caatinga no estado de Pernambuco. Em estudo realizado na Reserva da Biosfera Xérica do México, Estrada-Torres et al. (2009) referem a presença de Comatricha pulchella apenas no folhedo, seu microhábitat característico, sem nenhum registro nos numerosos cactos analisados. Considerando a constância e abundância nos substratos analisados, Comatricha pulchella mostrou-se acidental e escassa, esporulando na estiagem (Tabela 1).

A mixobiota registrada nas cactáceas analisadas evidenciou elevada diversidade taxonômica $(\mathrm{S} / \mathrm{G}=$ 0,63-1,5), particularmente no mandacaru, mostrando que este microhábitat apresenta grande potencial para desenvolvimento dos mixomicetos. Estrada-Torres et al. (2009) também observaram elevada diversidade taxonômica ao analisarem a mixobiota associada a cactos e outras plantas suculentas do Vale Tehuacán-Cuicatlán, zona árida do México.

Mais da metade das espécies registradas no presente estudo foi considerada como acidental e escassa na mixobiota local, esporulando preferencialmente durante a estiagem.

Dentre as quatro ordens registradas nas cactáceas analisadas, Physarales foi a mais representativa,

TABELA 1: Constância, abundância, substrato de esporulação e sazonalidade das espécies de Myxomycetes encontradas nos três municípios do agreste e sertão de Pernambuco. M- mandacaru; X-xique-xique; P- palma forrageira; C- coroa de frade; ES- estiagem; $\mathrm{CH}$ - chuvoso. Abundância - escassa $(<1,5 \%)$, ocasional $(1,5-3,5 \%)$, comum $(>3,5-6,5 \%)$, abundante $(>6,5 \%)$; Constância - acidental $(<25 \%)$, acessória $(25-50 \%)$, constante $(>50 \%)$.

\begin{tabular}{lccccc}
\hline \multicolumn{1}{c}{ Espécie } & $\begin{array}{c}\text { Constância } \\
(\%)\end{array}$ & $\begin{array}{c}\text { Abundância } \\
(\mathbf{\%})\end{array}$ & Substrato & \multicolumn{2}{c}{ Espécimes (no) } \\
ES & CH \\
\hline Arcyria cinerea & 8,3 & 1,1 & $\mathrm{M}, \mathrm{P}$ & 2 & - \\
Badhamia melanospora & 66,7 & 47,1 & $\mathrm{M}, \mathrm{X}, \mathrm{P}, \mathrm{C}$ & 52 & 25 \\
Ceratiomyxa fruticulosa & 25 & 1,6 & $\mathrm{M}$ & 3 & - \\
Comatricha pulchella & 8,3 & 1,1 & $\mathrm{M}$ & 2 & - \\
Diachea leucopodia & 8,3 & 0,5 & $\mathrm{X}$ & 1 & - \\
Didymium minus & 8,3 & 0,5 & $\mathrm{X}$ & 1 & - \\
Didymium nigripes & 8,3 & 0,5 & $\mathrm{X}$ & 1 & - \\
Didymium squamulosum & 8,3 & 2,7 & $\mathrm{P}$ & 5 & - \\
Hemitrichia calyculata & 8,3 & 0,5 & $\mathrm{M}$ & 1 & - \\
Hemitrichia serpula & 8,3 & 0,5 & $\mathrm{M}$ & 1 & - \\
Perichaena corticalis & 8,3 & 2,7 & $\mathrm{C}$ & 5 & - \\
Perichaena depressa & 16,7 & 1,6 & $\mathrm{M}$ & 3 & - \\
Physarum bogoriense & 16,7 & 1,6 & $\mathrm{M}$ & - & 3 \\
Physarum compressum & 58,3 & 33,5 & $\mathrm{M}, \mathrm{X}, \mathrm{P}, \mathrm{C}$ & 42 & 20 \\
Physarum echinosporum & 25 & 2,2 & $\mathrm{M}, \mathrm{P}$ & - & 4 \\
Physarum pusillum & 8,3 & 2,2 & $\mathrm{M}, \mathrm{X}, \mathrm{P}$ & - & 4 \\
\hline
\end{tabular}


correspondendo aproximadamente a $90 \%$ do total das coleções (Figura 2). Essa predominância também foi observada em outras regiões áridas do planeta (SCHNITTLER; NOVOZHILOV, 2000; NOVOZHILOV et al., 2006; LADO et al., 2006), sugerindo que as espécies dessa ordem possam suportar mais facilmente que as outras a dessecação do substrato. Badhamia melanospora e Physarum compressum (Physaraceae) caracterizam a mixobiota suculentícola do ambiente estudado, pela sua constância e abundância.

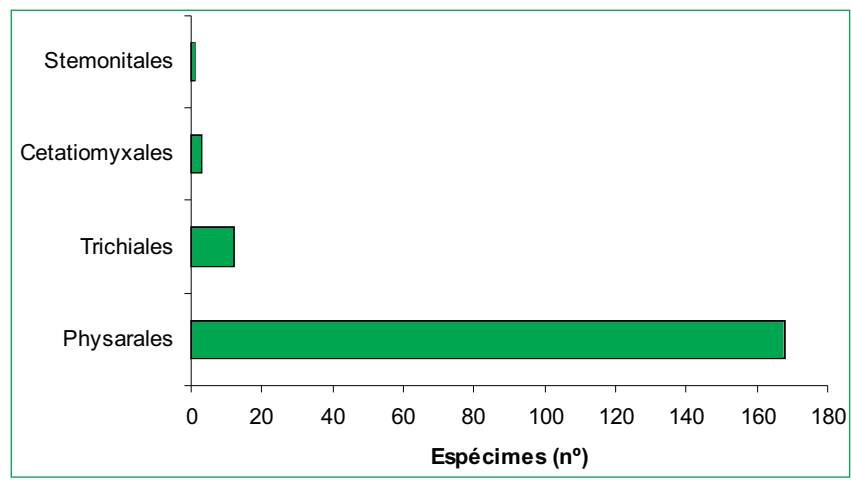

FIGURA 2: Representatividade das ordens de Myxomycetes encontradas sobre Cactaceae em três municípios do agreste e sertão pernambucano.

Badhamia melanospora ocorre de forma significativa em ambientes áridos e semi-áridos, presente em substratos que possuem boa capacidade de retenção hídrica, principalmente cactos (NOVOZHILOV et al., 2003; ESTRADA-TORRES et al., 2009; LADO et al. 2006). Em estudo desenvolvido por Lado et al. (2006) em áreas desérticas do Chile, Badhamia melanospora foi registrada no ponto mais seco do deserto, onde nenhum outro mixomiceto foi encontrado. No presente estudo, o faneroplasmódio de Badhamia melanospora originou numerosos esporocarpos, tanto no período mais úmido quanto naquele mais seco.

\section{Agradecimentos}

Os autores agradecem ao Conselho Nacional de Desenvolvimento Científico e Tecnológico (CNPq), pela concessão de financiamento (Proc. 485571/2006-4) e bolsa de produtividade em pesquisa à L. H. Cavalcanti; à Isadora Lima Coelho, Leandro de A. N. N. Agra, Glauciane Damasceno, Juciara Carneiro Gouveia Tenório, David Barreiro Nunes Lemos, Maria de Fátima de Andrade Bezerra, Márcio Ulisses de Lima Rufino e ao Sr. Gilcean Jones da Silva, pela ajuda nas coletas e auxílio na parte técnica deste trabalho.

\section{Referências}

AB'SABER, A. N. Ecossistemas do Brasil. São Paulo: Metalivros. 2008. 100 p.

ALVES, M. H.; COSTA, A. A. A.; CAVAlCANTI, L. H. Myxomycetes, state of Ceará, northeastern Brazil. Check List, Rio Claro, v. 6, n. 4, p. 555-558, 2010.

BELTRÁN-TEJERA, E.; MOSQUERA, J.; LADO, C. Myxomycete diversity from arid and semiarid zones of the Canary Islands (Spain). Mycotaxon, Ithaca, v. 113. p. 439-442, 2010.

BEZERRA, A. C. C.; CAVAlCANTI, L. H.; DIANESE, J. C. Species of Hemitrichia (Trichiaceae, Myxomycetes) in Brazil. Mycotaxon, Ithaca, v. 107, p. 35-48, 2009.

CASTIllo, A. C.; ILlANA, C.; MORENO, G. Badhamia melanospora Speg. a species wrongly interpreted. Mycotaxon, Ithaca, v. 7, p. 163-170, 1996.

CAVALCANTI, L. H. Biodiversidade e distribuição de mixomicetos em ambientes naturais e antropogênicos no Brasil: espécies ocorrentes nas Regiões Norte e Nordeste. In: ARAÚJO, E. L.; MOURA, A. N.; SAMPAIO, E. V. S. B.; GESTINARI, L. M. S.; CARNEIRO, J. M. T. (Eds). Biodiversidade, conservação e uso sustentável da flora do Brasil. Recife: Sociedade Botânica do Brasil, 2002. p. 209-216.

CAVAlCANTI, L. H.; BEZERRA, A. C. C.; COSTA, A. A. A.; FERREIRA, I. N.; BEZERRA, M. F. A. Distribution of Diachea (Didymiaceae, Myxomycetes) in the northeastern region of Brazil. Mycotaxon, Ithaca, v. 110, p. 163-172, 2009.

CAVALCANTI, L. H.; MOBIN, M. Myxomycetes associated with palm trees at the Sete Cidades National Park, Piauí State, Brazil. Systematics and geography of plants, Meise, v. 74, p. 109-127, 2004.

CAVAlCANTI, L. H.; SOUZA, W. P.; SANTOS, D. S.; GÓESNETO, A. Filo Myxomycota. In: GUSMÃO, L. F. P.; MAIA, L. C. (Eds). Diversidade e caracterização dos fungos do semiárido brasileiro. V.II. Recife: Associação de Plantas do Nordeste, 2006. p. 49-74.

COSTA, A. A. A.; FERREIRA, I. N.; BEZERRA, M. F. A.; CAVALCANTI, L. H. Mixobiota de Floresta Atlântica: novas referências de Physarales para o estado da Paraíba, Nordeste do Brasil. Revista Brasileira de Botânica, São Paulo, 2011 (no prelo). ESTRADA-TORRES, A.; WRIGLEY DE BASANTA, D.; CONDE, E.; LADO, C. Myxomycetes associated with dryland ecosystems of the Tehuacán-Cuicatlán Valley Biosphere Reserve, Mexico. Fungal Diversity, Chiang Mai, v. 36, p. 17-56, 2009.

FARR, M. L. Flora Neotropica. Monograph 16. New York: New York Botanical Garden, USA, 1976. 304 p.

GotTsBerger, G. Myxomyceten aus Bahia und Goiás. Nova Hedwigia, Stuttgart, v.15, p. 361-368, 1968.

GUSMÃO, L. F. P.; GÓES-NETO, A.; CRUZ, A. C. R. Fungos. In: JUNCÁ, F. A.; FUNCH, L.; ROCHA, W. (Org.). Biodiversidade 
e conservação da Chapada Diamantina. Brasília: Ministério do Meio Ambiente, Serie Biodiversidade. 2005. p. 233-240.

GUSMÃO, L. F. P.; MAIA, L. C. (Org.). Diversidade e caracterização dos fungos do semiárido brasileiro. Recife: Associação de Plantas do Nordeste (APNE), v. II. 2006. p. 27-47.

JATOBÁ, L. Vegetação de Pernambuco. In: ANDRADE, M. C. O. (Coord.). Atlas Escolar Pernambuco. João Pessoa: Grafset, 2003. p. 59-62.

LADO, C. Nomenmyx. Anomenclatural Taxabase of Myxomycetes. Cuadernos de Trabajo de Flora Micológica Ibérica16. Consejo Superior de Investigaciones Científicas (CSIC). Madrid: Real Jardín Botánico, 2001. 219 p.

LADO, C.; ESTRADA-TORRES, A.; STEPHENSON, S. L. Myxomycetes collected in the first phase of a north-south transect of Chile. Fungal Diversity, Chiang Mai, v. 25, n. 30, p. 81-101, 2006.

LADO, C.; PANDO, F. Flora Mycologica Ibérica. v. 2. Myxomycetes I. Ceratiomyxales, Echinosteliales, Liceales, Trichiales. Consejo Superior de Investigaciones Científicas (CSIC). Madrid: Real Jardín Botánico, 1997. 323 p.

LEAL, I. R.; TABARELLI, M.; SILVA, J. M. C. da (Ed.). Ecologia e conservação da caatinga. Recife: Universidade Federal de Pernambuco, 2003. $804 \mathrm{p}$.

LISTER, A. A monograph of the Mycetozoa. London: British Museum Natural History, 1925. 222 p.

MAIMONI-RODELLA, R. C. S. Biodiversidade e distribuição de mixomicetos em ambientes naturais e antropogênicos no Brasil: regiões Sudeste e Centro-oeste. In: ARAÚJO, E. L.; MOURA, A N.; SAMPAIO, E. V. S. B.; GESTINARI, L. M. S.; CARNEIRO, J. M. T. (Ed.). Biodiversidade, conservação e uso sustentável da flora do Brasil. Recife: Universidade Federal Rural de Pernambuco, Sociedade Botânica do Brasil, 2002. p. 217-220.

MARTIN, G. W.; AleXOPOUlOS, C. J. The Myxomycetes. Iowa City: University of Iowa Press. 1969. 560 p.

MARTIN, G. W.; ALEXOPOULOS, C. J.; FARR, M. L. The genera of Myxomycetes. Iowa City: University of Iowa Press, $1983.560 \mathrm{p}$.

MOSQUERA, J.; LADO, C.; ESTRADA-TORRES, A.; BELTRÁN TEJERA, E. Trichia perichaenoides, a new Myxomycete associated with decaying succulent plants. Mycotaxon, Ithaca, v. 75, p. 319328,2000

MOSQUERA, J.; LADO, C.; ESTRADA-TORRES, A.; BELTRÁN TEJERA, E. Description and culture of a new Myxomycete, Licea succulenticola. Anales Jardín Botánico de Madrid, Madrid, v. 60, p. 3-10, 2003.

NOVOZHILOV, Y. K.; MITCHELL, D. W.; SCHNITTLER, M. Myxomycete biodiversity of the Colorado Plateau. Mycological Progress, München, v. 2, p. 243-258, 2003.

NOVOZHILOV, Y. K.; ZENLYANSKAYA I. V.; SCHNITTLER, M. T. Two new species of Perichaena (Myxomycetes) from arid areas of Russia and Kazakhstan. Mycologia, Stanford, v. 100, p. 816-822, 2008.
NOVOZHILOV, Y. K.; ZEMLIANSKAIA, I. V.; SCHNITTLER, M.; STEPHENSON, S. L. Myxomycete diversity and ecology in the arid regions of the Lower Volga River Basin (Russia). Fungal Diversity, Chiang Mai, v. 23, p. 193-241, 2006.

PINHEIRO, K.; ALVES, M. Espécies arbóreas de uma área de Caatinga no sertão de Pernambuco, Brasil: dados preliminares. Revista Brasileira de Biociências, Porto Alegre, v. 5, n. 2, p. 426428, 2007

RODAL, M. J. N.; COSTA, K. C. C; LINS-E-SILVA, A. C. B.; FERNANDES, C. H. M.; LIMA, A. L. A. de; SILVA, M. C. N. A. Flora vascular e formas de vida das plantas em um hectare de vegetação de caatinga no nordeste brasileiro. Agrária, Recife, v. 4, p. 17-24, 2009.

RODAL, M. J. N.; MARTINS, F. R.; SAMPAIO, E. V. S. B. Levantamento quantitativo das plantas lenhosas em trechos de vegetação de caatinga em Pernambuco. Caatinga, Mossoró, v. 21, p. 192-2005, 2008.

SAMPAIO, E.V.S.B; GIULIETTI, A.M.; VIRGÍNIO, J.; GAMARRA-ROJAS, C. 2002. Vegetação e Flora da Caatinga. Recife, Associação de Plantas do Nordeste (APNE) e Centro Nordestino de Informações sobre Plantas (CNIP).

SCHNITTLER, M. Ecology of Myxomycetes of a winter-cold desert in Western Kazakhstan. Mycologia, Stanford, v. 93, n. 4, p. 653-669, 2001.

SCHNITTLER, M.; STEPHENSON, S. L. Inflorescences of Neotropical herbs as a newly discovered microhabitat for Myxomycetes. Mycologia, Stanford, v. 94, n. 1, p. 6-20, 2002.

SCHNITTLER, M.; LADO, C.; STEPHENSON, S. L. Rapid biodiversity assessment of a tropical myxomycete assemblage Maquipucuna Cloud Forest Reserve, Ecuador. Fungal Diversity, Chiang Mai ,v. 9, p. 135-167, 2002.

SCHNITTLER, M.; NOVOZHILOV, Y. Myxomycetes of the winter-cold desert in Western Kazakhistan. Mycotaxon, Ithaca, v. 74 , p. 267-285, 2000

SPEGAZZINI, C. Fungi Argentini. Pugillus tertius. Anales Sociedad Científica Argentina, Buenos Aires, v. 10, p. 145-168, 1880.

STEPHENSON, S. L. Distribution and ecology of myxomycetes in temperate forests. II. Patterns of occurrence on bark surface of living trees, leaf litter, and dung. Mycologia, Stanford, v. 81, p. 608-621, 1989.

STEPHENSON, S. L.; KALYANASUNDARAM, I.; LAKHANPAL, T. N. A comparative biogeographical study of myxomycetes in the mid-Appalachian of eastern North America and two regions of India. Journal of Biogeography, San Francisco, v. 20, p. 645-657, 1993.

SUDENE. Dados pluviométricos mensais do Nordeste. Recife: Superintendência do Desenvolvimento do Nordeste, 1990. 50 p. 